II. Ans dem Allerheiligen-Hospital zu Breslau, Abtheilung des Hern Prof. Dr. Rosenbach.

\title{
Zur Lehre von der infectiösen fieberhaften,
} mit Icterus complicirten Gastroenteritis (Weil'sche Krankheit).

Von Dr. Otto Stirl, Volontärarzt.

Seitdem Weil auf eine eigenthümliche, mit Milztumor, Icterus und Nephritis einhergehende acute Infectionskrankheit aufmerksam gemacht hat, sind von verschiedenen Seiten ähnliche Beobachtungen veröffentlicht worden. Nichtsdestoweniger ist die Frage von der Weil'schen Krankheit durchaus noch nicht gelöst; im Gegentheil, je zahlreicher die Fälle waren, um so mehr differirten die Ansichten der Autoren von einander. Einig sind sie nur darüber, dass die genannte Krankheit eine acute Infectionskrankheit sei. Dagegen herrscht schon über die Natur dieser Infectionskrankheit eine grosse Mannichfaltigkeit der Ansichteu. Während Weil selbst die Frage unentschieden lässt, ob die Krankheit ein Morbus sui generis oder uuter die bekannten Infectionskrankheiten zu rechnen sei, treten andere Autoren mit Entschiedenheit für diese, andere für jene Auffassung ein. Wieder andere sprechen der sogenannten Weil'schen Krankheit jede anatomische, ätiologische und symptomatologische Einheit ab. 
Eine ähnliche Uneinigkeit herrscht betreffs der Symptome der Krankheit. Unbestritten sind der fieberhafte Icterus, die gastrischen und die cerebralen Störungen. Ueber den Verlauf des Fiebers besteht nur insofern eine Einigkeit, als ein meist plötzliches Ansteigen und lytischer Abfall von allen Seiten zugegeben wird. Das Recidiv aber, das von Weil für eine charakteristische Eigenthümliclıkeit der Fiebercurve erklärt wird, ist von vielen Autoren nicht beobachtet worden; auch die Wagner'sche Deutung, dass dasselbe nur bei den schwereren Fällen auftrete, wird von Fiedler bestritten, da derselbe das Recidiv bei mehreren schweren Fällen gerade vermisste. Kirchner lässt es dahingestellt sein, ob der in seinen Fällen wiederholt beobachtete Relaps auf Rechnung von zufälligen Complicationen zu setzen sei, wällrend Ha as geneigt ist, lierfür Diätfehler verantwortlich zu machen. - Verschieden ist ferner die Auffassung betreffs der Betheiligung von Milz uud Nieren. Die ıneisten Autoren zwar bestätigen die Beobachtungen Weil's, dass von vornherein Milztumor und Nephritis auftreten, andere aber, namentlich Fiedler, bestreiten die Regelmässigkeit dieser Symptome. Dagegen legt dieser Autor grosses Gewicht auf die Muskelschmerzen, namentlich in den Waden, die er direkt für pathognomonisch erklärt.

Endlich erblickt Hueber in der acut entzündlichen Affection der gesammten Schleimhäute auf der Höhe des Krankheitsprocesses ein charakteristisches Merkmal der Krankhcit, eine Ansicht, die von keinem anderen Autor getheilt wird.

1) jese Streitfrage nun, wenigstens in einem gewissen Simne zu lösen, sind wir an der Hand eines Falles in der Lage, der auf der Abtheilung des Herrn Prof. Rosenbach zu Breslau zur Beobacltung gelangte. Namentlich scheint der Fall geeiguet, über die Aetiologie der Krankheit Klarheit zu bringen; weshalb el uns zur Veröffentlichung geeignet erscheint.

Aus der Krankengeschichte heben wir folgendes hervor:

Pat., der 35 jährige Canalarbeiter H., giebt an, früher gesund gewesen zu sein. Im Juni vorigen Jahlres verunglückte er in der Weise, dass er von den aufsteigenden Cloakengasen betäubt in die Jauche stürzte und dabei eine grosse Quantität derselben verschluckte. Herausgezogen, erbrach er den grössten Theil wieder, doch lag er im Anschluss daran mehrere Stunden im Schüttelfrost Neben Störung des Allgemeinbefindens bestanden in den nächsten Tagen Erbrechen und Diarrlüen, die ihn schliesslich zwangen, die Arbeit einzustellen und das Bett aufzusuchen.

Gleichzeitig mit ibm verunglückte eill zweitel Arbeiter in derselben Weise; auch dieser erkrankts nach unserer Ermittelung unter den gleichen Symptomen.

Bei der Aufnahme in's Hospital zeigte Pat., der ein kräftig gebauter Mann mit gut entwickelter Muskulatur und starkem Fettpolster war, folgende Symptome:

Die Temperatur betrug 39,3; dieselbe fiel am nächsten Tage spontan zur Norm, um am darauf folgenden wieder bis auf 40,1 zu steigen; von da an fiel sie Irtisch, und zwar staffeiförmig ab und erreichte nach 6 Tagen die Norm; spätcr war sie einle Zeit lang selbst subnormal. Doch blieb Pat. nicht fortdauernd fieberfrei; am 24. Beobachtungstage nämlich stieg die Temperatur wieder auf 38,3 , um am nächsten Tage wieder zur Norm zurückzukehren.

Der Leib war aufgetriebell, die Leber vergrössert und druckempfinclich. Am 3. Beobachtungstage zeigten die Hant und die Conjunctivia eine icterische Färbung; auffallend war, dass am gleichen Tage die lytische Defervescenz begann. Die Milz war deutlich vergrössert. Der Urin, del in den ersten Tagen der Erkrankung keine abnormen Erscheinungen darbot, war später eiweisshaltio und icterisch gefärbt; mikroskopisclı waren Cylinder und rothe und weisse Blutzörperchen nachweisbar.

Von cerebralen Erscheinungen waren Kopfschmerzell, Schwindel, Benommenheit und selbst Delírien vorhanden; elnmal drohte Pat. einem Collaps 'zu' erliegen aus dem er nur durch starke Stimulantien wieder erweckt werden konnte. Ausserdem stellten sich im späteren Stadium der Erkrankung Parese der beiden Oberextremităten, der linken Augenmuskulatur und des linken Facialis ein; der Hintergrund beider Augen bot das Bild der Staungspapille. Das anfängliche Erbrechen und die Diarrhöen bestanden auch späte noch, ebenso wie Appetitlosigkeit; die Stülle waren, wenn auch schwach, so doch stcts gallig gefärbt. Der Leib wal' aufgetrieben und druckempfindlich, namentlich in der Ileocöcalgegend. - Während von seiten der Lungen aussel geringer Bronchítis keine Symptome vollagen, war die Herzdïmpfung nach beiden Seiten hin verbreitert; die Herztöne waron dumpf, und ausserdem war ein Reiben und Scliwirren hörbar, das über dəm Ansatz der dritten linken Rippe am stärksten war.

Die Haut des Abdomen zeigte rötbliche Stellen, die man abor nicht mit Sicherheit als Roseola ansprechen konnte; die Schleimhaut der Lippen und des Mundes war mit Borken besetzt, die von Blutungen herrührten. Gegen Ende der Krankheit traten spontaner Krampf der Wadenmuskulatur und Schmerzhaftigkeit bei Berührung auf, die aber bald wieder nachliessen.

Die Krankbeit selbst nahm ihren Ausgang in Heilung, wenngleich die Recouvalescenz eine auffallend langsame war. Was den zweiten oben erwähnten Fall betrifft, der leiłer nicht unter unserer Beobachtung verlief, so hörten wir, dass die Krankheit, deren auffallendstes Symptonn der Icterus war, letal verlief.

Ueberblicken wir nun den Symptomencomplex, so finden wir, dass sich unser Fall den Weil'schen Fällen eng anschliesst, so dass an ihrer Identität kaum gezweifelt werden kann. Aucl wir haben es mit einem kräftigen Mann in deu mittleren Jahren zu thun, der zul. Sommerszeit eine acute Krankheit acquirirt, deren Hauptsymptorne fieberhafter Icterus, Milztumor und Nephritis sind. Als einziges ätiologisches Moment kann in unzweifelhafter Weise nur das Verschlucken von Jauche nachgewiesen werden, da der zweite el'wähnte Fall, der aus gleicher Ursache an den gleichen Symptomen erkrankte, gleichsam die Probe für das Exempel liefert.

Die Infection erfolgte also rom Verdauungstractus aus, und zwar scheint das Auftreten der Schüttelfröste auf eine schubweise Aufualıne der iufectiösen Schädlichkeiten zu deuten. Es bestätigt sich somit die von Fiedler, namentlich aber von $\mathrm{Kirchner}$ und Pf $a h_{l} l$ vertretene Ansicht, dass der Weg der Infection der Magendarmcanal sci; namentlich die beiden letzten, die kleine Epidemieen beobachteten, waren in der Lage, der Wahrheit am nächsten zu kommen, da sie durch Auffinden der die Gesammtheit treffenden Schädlichkeiten alle falschen subjectiven Vermuthungen ausschliessen konnten. So kamen bcide zu dem Schluss, dass das Baden in verunreinigtem Wasser und gelegentliches Verschlucken desselben ätiologisch anzuschuldigen sei.

Wenngleich nul unser Fall über den Weg del Infection Aufklärung zu bringen imstande ist, so vermag er das Dunkel, das über die Natur der Infectionsträger herrscht, nicht zu lichten. Die von Goldschmidt erwähnten Kurzstäbclsen, mit denen die Cylinder besetzt waren, konuten wir nicht fiudell. Ebenso wenig vermögen wir zu entscheiden, ob den von Brodowski und Dunin gefundenen Mikrokokken, oder den Bacillen, die $\mathrm{Nauwerck}$ in den nekrotischen Darmpartieen beobachtete, eine Bedeutung beizumessen ist, da uns kein Sectionsbefund vorliegt. Soviel scheint aber aus unseren Beobachtungen sicher hervorzugehen, dass es nämlich zum $\mathrm{Zu}$ standekommen der Iufection durchaus nicht des Einwanderns von Mikroorganismen bedarf; das unmittelbare Auftreten von Schüttelfrösten nach dem Unfall beweist, dass das Einführen von Ptomainen oder, was wahrscheinlicher ist, von anderen in der Jauche befindlichen Toxiıen genügt, um die Krankheit zum Ausbruch zu bringen. Wäre die Infection durch die Mikroorganismen selbst erfolgt, sn hätte bis zum Ausbruclı der Schüttelfröste eiue geraume Zeit verstreichen müssen, bis die Ptomaine, die doch heute allgemein als das toxisch wirkende Agens aufgefasst werden, producirt waren. Damit soll aber durchaus nicht die Möglichkeit ausgeschlossen werden, dass in auderen Fällen die Infection durch kleinste Organismen erfolgt im Gegentheil scheinen die Fälle, wo die Krankheit ein Prodromalstadium zeigte, diese Deutung zu verlangen; es bedurfte dann eben zum Auftreten del. Symptome erst einer Vermelıung der Organismen und der Bildung von Ptomainen.

Der Weg del Infection nun scheint auch das auffallendste Symptom der Krankheit, deıl Icterus, zu erklären. Nach den Untersuchungen Stadelmanu's sind es zwei Factoren, die bei gewissen Intoxicationen das Zustandekommen des Icterus bewirken; erstens wird eine selır zähe Galle secernirt, die vermöge ihrel Consistenz nur schwer instande ist, abzufliessen, und zweitens zeigen die Leberzellen die Erscheiuungen der trüben Schwellung uud drücken auf die Gallencapillaren, dadurch wird eine verstärkte Resorption veranlasst. Einell analogen Reizvorgang in der Leber bei unserer und anderen ähnlichen Intoxicationen anzunelımen, berechtigt uns nicht allein die fast stets nachweisbare Leberschwellung, soudern auch der von verscliedenen Autoren in übereinstimmender Weise angegebene Sectionsbefund; immer warcn neben Entzündungsherden im interstitiellen Biudegewebe albuminoide und fettige Eutartung des Leberparenchyms nachweisbar. Gelegentlich einer Besprechung unseres Falles äusserte Her Prof. Rosenbach, dass in diesen Factoren allein der Grund für das Zustandekommen des Icterus nicht $\mathrm{zu}$ suchen sei, was schon die gallige Färbung der Stülıle beweise; er glaubt vielmelır, dass die infectiösen Schädlichkeiten, seien es nun Orgauismen oder Ptomaine, welche direkt auf der Bahn des Pfortaderblutes oder iudirekt durch Vermittelung der Lymphbahnen und des Aortensystems in die Leber gelangen, dieselbe reizen und zu encrgischer Thätigkeit anregen. Infolge dieser andauernd hochgradig vermehrten Secretion hauptsächlich und vielleicht auch del oben erwähnten Stauung, namentlich wenn infolge verstärkter Darmperistaltik del. Druck im Dünndarm ein besonders holıer, und dadurch der Gallenabfluss erscliwert wird, werde die Galle in den Capillaren unter einen abnorm loheu Druck gesetzt, der dieselbe in fremde Bahnen, d. i. in die Lymplwwege treibe, so dass Icterus entstehe." Er glaubt ferner, dass die energischere Thätigkeit der Leber keine zwecklose sei, dass vielmehr in ihr das Bestreben des Körpers sich ausdrücke, die infectiösen Schädlichkeiten unschädlich zu machen. Betreffs der Art und Weise, wie dieser Zweck erreicht wird, wären zwei Möglichkeiten denkbar; entweder die zur Resorption gelangenden Bestandtheile selbst sind es, welche diesen Schädlichlieitell die Lebensbedingungen rauben bezw. ihre toxisch wirkendeu Producte paralysiren, oder aber die Leber vermag dies durch eine Erhöhung ihrer eigenen uns ihrem Wesen nach unbe- 
kannten Thätigkeit, vermöge welcher sie die Beschaffenheit des sie durchfliessenden Blutes regulirt, und als deren uns erkennbares Symptom eine Vermehrung des Lebersecretes, der Galle, die unter gewissen Umständen zum Icterus führen muss, zu Tage tritt. Im ersten Falle wäre also, kurz gesagt, der Icterus selbst, d. h. die Circulation von Gallenbestandtheilen im Blut, die Schutzmaassregel, im letzten versteckte sich hinter ihm nur eine besondere erhöhte Thätigkeit der Leber. Wenn, wie Voit behauptet, der Galle wirklich keine antiseptischen Eigenschaften innewohnen, so müsste an das letztere gedacht werden.

Jedenfalls drängt sich der Gedanke auf. dass es einen Schutzwall gegen die Infection vom Darmcanal aus gebe, wenn man überlegt, wie selten pyämische Zustände durch Invasion auf diesem Wege zu Stande kommen, obgleich die Gelegenheiten dazu so überaus mannichfaltig sind. Und dass dieser Schutzwall kein anderer als die Leber sein kann, beweist die häufige Combination einerseits von Icterus mit derartigen Infectionen, andererseits aber die geringe Intensität des Icterus bei Blutvergiftungen durch eine andere Pforte, als den Verdanungstractus; lierbei ist das Gift bereits in anderen Gebieten localisirt, so dass die Leber nicht mehr in genügende Thätigkeit tritt, oder sie ist durch die Stärke der den Organismus treffenden Schädlichkeiten so in Mitleidenschaft gezogen, dass sie eine energische Thätigkeit nicht mehr auszuïben vermag; daher kommt es bei schwerer Pyämie nur zu Spuren von Icterus. - So ist auch das Zustandekommen des sogenannten katarrhalischen Icterus zu erklären, so dass derselbe seiner Natur nach nicht mehr oder wenigstens nicht ausschliesslich als Stanungsicterus aufzufassen ist, sondern als eine Reaction der Leber auf Eindringen derselben Schädlichkeiten, die auch den Magendarmkatarrh veranlassten."

Diese Auffassung von dem Verhältniss des Icterus zur Infection scheint durch die auffallende Thatsache ihre Bestätigung zu finden, dass nicht allein in unserem Falle, sondern, soweit sich dies controlliren lässt, auch in den meisten anderen in der Litteratur vorhandenen Fällen das Auftreten des Icterus mit dem Beginn der Defervescenz zusammenfiel.

Schon Pfuhl betrachtete dieses Zusammentreffen nicht als eine zufällige Erscheinung, vielmehr vermuthete er, dass es sich hierbei um ein ursächliches Verbältniss handle.

Die Wechselwirkung nun zwischen Icterus und Fieber ist es nach Rosenbach, welche bestimmend auf den Verlauf der Infectionen vom Verdauungstractus einwirkt. Es sind in dieser Beziehung drei Möglichkeiten denkbar: 1) entweder vermag durch Erbrechen oder Diarrhöen der Darmcanal die infectiösen Schädlichkeiten selbst anszuschalten, so dass es der genannten Leberthätigkeit, die durch Icterus sich documentirt, nicht bedarf - ein Fall, welcher der denkbar günstigste ist und z. B. bei Cholera nostras eintritt, oder 2) die Leber vermag die Allgemeininfection zu verhindern, so dass Icterus ohne oder mit nur geringen Fiebererscheinungen auftritt, wie beim Icterus catarrhalis, oder 3) die Leber ist nicht imstande, dem Gift einen Damm zu setzen, dasselbe gelangt vielmehr in die Blutbahn, erregt Fieber, zugleich aber eine noch energischere Leberthätigkeit, vermöge deren der Körper der Infection entweder Herr zu werden vermag - daher mit Auftreten des Icterus Beginn der Defervescenz -; oder trotz der erhöhten Leberthätigkeit gelingt dies dem Körper auch jetzt noch nicht daher trotz Icterus kein Fieberabfall, sondern letaler Ausgang unter pyämischen Erscheinungen.

In diese letzte Kategorie nun sind die Fäle der Weil'schen Krankheit $\mathrm{zu}$ rechnen. Zwar scheint mit dieser Annahme die Behauptung Weil's im Widerspruch zu stehen, dass ein günstiger Ausgang fïr die Krankheit charakteristisch sei. Doch schon unsere Erfahrungen beweisen, dass dies durchaus nicht der Fall ist, dass der Ausgang vielmehr je nach der Schwere des Falles variirt, die ihrerseits wieder durch die Stärke des Giftes einerseits und der Resistenzfähigkeit des Individuums andererseits .bedingt wird. Jedenfalls war in unseren Fällen die Infection eine schwere, denn auch der schliesslich noch in Genesung ausgehende Fall drohte einem Collaps zu erliegen, wenn er nicht energisch stimulirend behandelt worden wäre.

Was also der sogenannten Weil'schen Krankheit ihr eigenthämliches Gepräge giebt, ist nach Rosenbach der Weg der Infection und der dadurch bedingte Icterus. Nur die Fälle sind daher zu dieser Krankheitsform zu rechnen, wo der Darm die Eingangspforte ist, alle anderen Infectionskrankheiten aber, die mit geringen Graden von Icterus complicirt sind, sind davon auszuschliessen. Namentlich ist der von A. Fränkel veröffentlichte Fall, wo die Symptome der Weil'schen Krankheit an eine Wundinfection sich anschlossen, zu dieser Krankheitsform nicht zu rechnen, da hier die Eingangspforte des Giftes eine andere war.

Die Weil'sche Krankheit ist daher nicht ein Morbus sui generis im Sinne der Autoren, sie ist vielmehr der specielle Fall einer Infection vom Verdauungstractus aus, wo die Intoxication gleichsam über die Leber hinweg erfolgt, nachträglich aber durch eine energische Thätigkeit derselben, die als Icterus in die Erschei. nung tritt, zu paralysiren gesucht wird. Der acute fieberhafte Icterus ist es also, der sie charakterisirt; all die anderen Symptome aber, wie Nephritis, Kopfschmerzen, Somnolenz, Muskelschmerzen u. s. w., sind durch die Allgemeininfection bedingt. Damit erklärt sich auch der auffallende Widerspruch, der unter den verschiedenen Autoren betreffs der Symptome herrscht; je nach der Schwere der Infection treten die Symptome in grösserer oder geringerer Intensität auf, je nach der Individualität des Falles tritt das eine oder das andere Symptom mehr in den Vordergrund.

Auch die weiteren cerebralen Symptome, die in unserem Falle beobachtet wurden, bestehend in Facialisparese, Augenmuskellähmung und Schwäche der oberen Extremitäten, könnte man von der Allgemeininfection herleiten; doch macht es die Beobachtung der Staungspapille wahrscheinlicher, dass dieselben durch erhöhten Hirndruck veranlasst wurden, der sich am ungezwungensten durch die Annahme eines Hämatoms der Dura mater - einer bei acuten Infectionskrankheiten nicht seltenen Erscheinung - erklären lässt.

Wollte man der Krankheit einen Namen geben, so wäre ,biliöses Typhoid" derjenige Name, der am präcisesten ihre Symptomatologie zu kennzeichnen geeignet ist. Doch knüpft sich an ihn zu sehr die Erinnerung an Typhus abdominalis, mit dem die Krankheit doch nichts gemein hat, als dass derselbe zu empfehlen wäre. Daher scheint uns die bereits oben gebrauchte Bezeichnung infectiös fieberhafte, mit Icterus complicirte Gastroenteritis" am besten das Wesen der Krankheit zu charakterisiren.

$$
\text { Lit teratur. }
$$

Weil, Ueber eine eigenthümliche mit Milztumor, Icterus und Nephritis einhergehende acute Infectionskrankheit. Deutsches Archiv f. klin. Med. Bd. 39. - A. Fränkel, Zur Lehre von der sogenannten We il'schen Krankheit. Deutsche med. Wochenschrift 1889, No.9. - Goldschmidt, Ein Beitrag zur neuen Infectionskrankheit Weil's. Deutsches Archiv f. klin. Med. Bd. 40. - Fiedler, Zur Weil'schen Krankheit. Ibid. Bd. 42. Hüeber, Die neue Infectionskrankleit Weil's in der Armee. Deutsche militärärztliche Zeitschrift 1888, p. 165. - Haas, Ein Beitrag zur neuen Infectionskrankheit Weil's. Prager medicin. Wochenschrift 1887, No. 39 bis 40. - Pfuhl, Typhus abdominalis mit Icterus. Deutsche militāräztl. Zeitschrift 1888, p. 385. - Wagner, 2 Fälle von fieberhaftem Icterus (Weil). Deutsches Archiv f. klin. Med. Bd. 40. - Nauwerck, Zur Kenntniss der fieberhaften Gelbsucht. IIünchener medicin. Wochenschrift 1888, No. 35. - Brodowski und Dunin, Ein Fall der sogenannten Weil'schen infectiōsen Krankheit. Deutsches Archiv f. klin. Med. Bd. 43. - Kirchner, Eine Epidemie von fieberhafter Gelbsucht. Deutsche militärärztl. Zeitschrift 1888, p. 193. - Stadelmann, Archiv für experimentelle Pathol. und Pharmak. Bd. XIV, XV, XVI. 